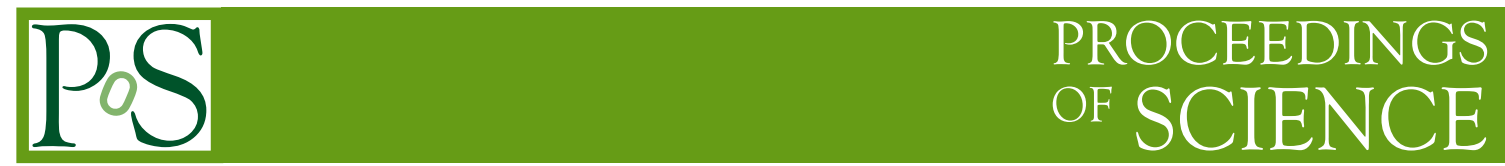

\title{
Fermion bag solutions to some sign problems in four-fermion field theories
}

\author{
Anyi Li* \\ Institute for Nuclear Theory, University of Washington, Seattle, 98195, USA \\ E-mail: anyiliauw.edu
}

Lattice four-fermion models containing $N$ flavors of staggered fermions, that are invariant under $Z_{2}$ and $U(1)$ chiral symmetries, are known to suffer from sign problems when formulated using the auxiliary field approach. Although these problems have been ignored in previous studies, they can be severe. In this talk, we show that the sign problems disappear when the models are formulated in the fermion bag approach, allowing us to solve them rigorously for the first time.

The 30 International Symposium on Lattice Field Theory - Lattice 2012,

June 24-29, 2012

Cairns, Australia

${ }^{*}$ Speaker. 


\section{Introduction}

Four-fermion field theories are interesting in both condensed matter and particle physics. The well known Hubbard model and its variants are often used in studying cuprate superconductors [1], antiferromagnets [2]. Low energy nuclear physics is also studied with four-fermion couplings in the effective field theory framework [3, 4]. In the context of more fundamental theories like QCD, four-fermion field theories offer a simpler setting to study phenomena like fermion mass generation and chiral symmetry breaking [5]. It has been suggested recently that quantum critical phenomena in graphene can be studied with four-fermion field theories [6, 7]. It has also been found there is a QCD-like sign problem in the four-fermion field theory [8]. Despite the wide interest, strongly coupled four-fermion field theories remain poorly understood as compared to their bosonic counterparts due to computational difficulties.

The only available method to compute quantities in a strongly interacting field theory with no small parameter is the Monte Carlo (MC) method. The traditional MC approach is to integrate the fermions out in favor of a determinant of a large fermion matrix, whenever this determinant is positive a non-local probability distribution emerges, which can be used to construct a MC method. Unfortunately, small eigenvalues of the fermion matrix which naturally arise in the presence of massless fermions can cause singularities in the traditional MC approach. This makes it difficult to study quantum critical phenomena containing massless fermions. In cases where the determinant of the fermion matrix is not positive, the original theory is said to suffer from a sign problem and the traditional approach is not useful. The repulsive Hubbard model away from half filling is a classic example where progress has been limited due to sign problems. Other relativistic four-fermion field theories like the Gross-Neveu (GN) models and Nambu-Jona-Lasinio (NJL) models are also known to suffer from sign problems in three or more space-time dimensions [9].

Recently a new approach called the fermion bag approach was proposed to solve some fourfermion field theories [10, 11, 12, 13, 14]. It is an extension of the meron cluster idea proposed some time ago [15]. The idea behind the fermion bag is to identify fermion degrees of freedom that cause sign problems and collect them in a bag and sum only over them. This is in contrast to traditional approaches where all fermion degrees of freedom in the entire thermodynamic volume are summed to solve the sign problem. In this talk, we consider lattice GN models containing $N$ flavors of massless staggered fermions with either a $Z_{2}$ or a $U(1)$ chiral symmetry [9]. While we work in three space-time dimensions, our results can easily be extended to higher dimensions. The $Z_{2}$ models with odd $N$ and all the $U(1)$ models are known to suffer from a sign problem when formulated in the traditional auxiliary field approach. Here we show that the sign problems disappear in the fermion bag approach.

\section{Auxiliary field approach}

Lattice GN models are formulated in the auxiliary field approach through the action

$$
S_{G N}=\sum_{x, y, i} \bar{\chi}_{i}(x)(D[\bar{\phi}])_{x, y} \chi_{i}(y)+S_{A F}
$$

where $\bar{\chi}_{i}(x), \chi_{i}(x)$ denote the Grassmann valued fermion fields of flavor $i=1,2 . ., N$ at the lattice site $x$. The explicit form of the auxiliary field action $S_{A F}$ depends on the GN model and will be 
discussed below. The matrix $D[\bar{\phi}]$ is defined by

$$
(D[\bar{\phi}])_{x y}=D_{x y}+\delta_{x y} \bar{\phi}(x),
$$

where $\bar{\phi}(x)$ is a function of the auxiliary fields as defined below and $D_{x, y}$ is the free staggered fermion matrixs,

$$
D_{x, y}=m \delta_{x, y}+\sum_{\alpha=1,2,3} \frac{\eta_{x, \alpha}}{2}\left[\delta_{x+\alpha, y}-\delta_{x, y+\alpha}\right] .
$$

Since we work in three dimensions, $\alpha$ labels the three directions, $\eta_{x, \alpha}=e^{\left(i \pi \zeta_{a} \cdot x\right)}, \zeta_{1}=(0,0,0)$, $\zeta_{2}=(1,0,0), \zeta_{3}=(1,1,0)$ are the staggered fermion phase factors and $m$ is the bare fermion mass. We assume anti-periodic boundary conditions in all directions and denote the lattice volume by $V=L^{3}$.

Following [9], we define the auxiliary fields on dual sites $\tilde{x}$. The model with a $Z_{2}$ chiral symmetry is defined through a single real auxiliary field $\sigma(\tilde{x})$, such that

$$
\begin{aligned}
S_{A F}[\sigma] & =\frac{N}{2 g^{2}} \sum_{\tilde{x}} \sigma^{2}(\tilde{x}), \\
\bar{\phi}(x) & =\frac{1}{8} \sum_{\langle\tilde{x}, x\rangle} \sigma(\tilde{x})
\end{aligned}
$$

while the model with a $U(1)$ chiral symmetry requires two real auxiliary fields $\sigma(\tilde{x})$ and $\pi(\tilde{x})$, such that

$$
\begin{aligned}
S_{A F}[\sigma, \pi] & =\frac{N}{4 g^{2}} \sum_{\tilde{x}}\left(\sigma^{2}(\tilde{x})+\pi^{2}(\tilde{x})\right), \\
\bar{\phi}(x) & =\frac{1}{8} \sum_{\langle\tilde{x}, x\rangle}(\sigma(\tilde{x})+i \varepsilon(x) \pi(\tilde{x})),
\end{aligned}
$$

where $\varepsilon(x)$ is the parity of a lattice site ( 1 on even sites and -1 on odd sites). In the above expressions, the set of nearest dual sites $\tilde{x}$ surrounding the fixed lattice site $x$ is denoted as $\langle\tilde{x}, x\rangle$. In this work we only consider these two classes of models.

The models contain a quantum critical point (QCP) separating a chirally symmetric phase (at small couplings) from a phase where the chiral symmetry is spontaneously broken (at large couplings). The symmetries that govern the QCP needs proper analysis due to fermion doubling. Without such an analysis it is difficult to establish the continuum field theory that emerges at the critical point [12]. In the traditional MC approach, one integrates over the Grassmann fields and writes the partition function of the GN models as

$$
\begin{aligned}
Z_{Z_{2}} & =\int[\mathscr{D} \sigma] e^{-S_{A F}[\sigma]}\{\operatorname{Det} D([\bar{\phi}])\}^{N}, \\
Z_{U(1)} & =\int[\mathscr{D} \sigma \mathscr{D} \pi] e^{-S_{A F}[\sigma, \pi]}\{\operatorname{Det} D([\bar{\phi}])\}^{N},
\end{aligned}
$$



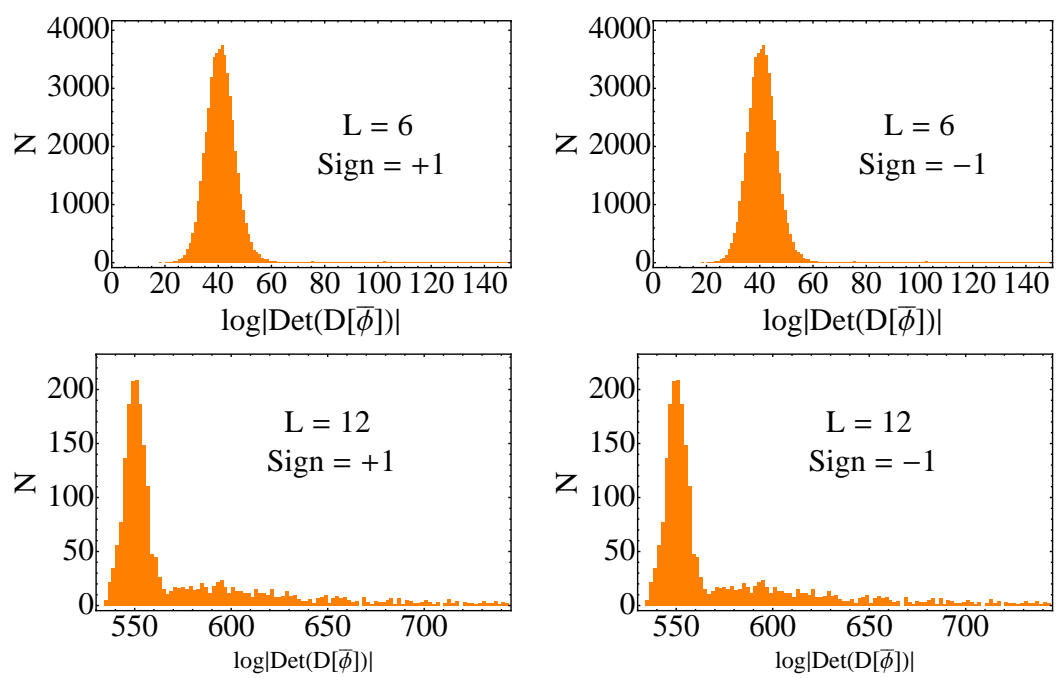

Figure 1: Distributions of positive (left graphs) and negative (right graphs) weight configurations as a function of $\log |\operatorname{Det}(D[\bar{\phi}])|$. One million configurations and 5000 configurations were generated at $6^{3}$ and $12^{3}$ lattices respectively. The distribution of positive configurations is almost identical to the one with negative configurations suggesting a severe sign problem.

In order to design a MC method the determinant terms in the above expressions have to be real and positive. In the $Z_{2}$ model since $\bar{\phi}$ is real, the matrix elements of $D[\bar{\phi}]$ are real. Hence, the determinant is real but not necessarily positive. In the case of the $U(1)$ model, $\bar{\phi}$ is complex and so the matrix elements of $D[\bar{\phi}]$ and its determinant can be complex. Hence, the $Z_{2}$ model as formulated in Eq. (2.8) suffers from a sign problem for all odd values of $N$, while the $U(1)$ model as formulated through Eq. (2.9) suffers from a sign problem for all values of $N$. In Fig. 1 we plot the distribution of configurations with positive and negative determinants as a function of $\log |\operatorname{Det}(D[\bar{\phi}])|$ for $6^{3}$ and $12^{3}$ lattices. As can be seen, the distribution of configurations with positive and negative weights are almost identical suggesting a severe sign problem rather than a mild one! Although we are not performing important sampling, our results clearly show that the sign problem must be studied carefully.

\section{Fermion bag approach}

We will now show that the sign problems in both the $Z_{2}$ and the $U(1)$ models discussed in section 2, disappear in the fermion bag approach. The proof relies on the fact that any $k_{i}$-point correlation function involving the $i^{\text {th }}$ flavor of staggered fermions defined through

$$
C_{i}\left(x_{i_{1}}, \ldots, x_{i_{k_{i}}}\right)=\int\left[d \bar{\chi}_{i} d \chi_{i}\right] \mathrm{e}^{-\sum_{x, y} \bar{\chi}_{i}(x) D_{x y} \chi_{i}(y)} \bar{\chi}_{i}\left(x_{i_{1}}\right) \chi_{i}\left(x_{i_{1}}\right) \ldots \bar{\chi}_{i}\left(x_{i_{k_{i}}}\right) \chi_{i}\left(x_{i_{k_{i}}}\right)
$$

is positive semi-definite. This is due to the special properties of the free staggered fermion matrix. Indeed, using the ideas developed in the fermion bag approach [12], we can write

$$
C_{i}\left(x_{i_{1}}, . ., x_{i_{k_{i}}}\right)=\operatorname{Det}(D) \operatorname{Det}\left(G\left[\{x\}_{i}\right]\right)=\operatorname{Det}\left(W\left[\{x\}_{i}\right]\right)
$$

where $G\left[\{x\}_{i}\right]$ is the $k_{i} \times k_{i}$ matrix of propagators between the $k_{i}$ sites in the set $\{x\}_{i} \equiv x_{i_{p}}, p=$ $1, \ldots, k_{i}$ whose matrix elements are $G_{x_{p}, x_{q}}=D_{x_{p}, x_{q}}^{-1}$ and the matrix $W\left[\{x\}_{i}\right]$ is a $\left(V-k_{i}\right) \times\left(V-k_{i}\right)$ 
matrix identical to the matrix $D$ except that the sites in the set $\{x\}_{i}$ are dropped from the matrix. All the determinants appearing in Eq.(3.2) can be shown to be positive (or zero). The simplest way to see this is to consider the matrix $W$. Since it is exactly the same as the staggered fermion matrix with some sites removed, its eigenvalues come in complex conjugate pairs of the form $m \pm i \lambda$. Unpaired eigenvalues are always $m$ and they too come in pairs when the lattice is bipartite. When $m=0$ then the determinant can be exactly zero. Thus, $C_{i}\left(x_{i_{1}}, . ., x_{i_{k_{i}}}\right) \geq 0$. We will use this property to prove the absence of a sign problem in the fermion bag approach.

Instead of integrating out the fermion fields let us integrate out the auxiliary fields first and construct the appropriate four fermion action for the models. Let us first consider the $Z_{2}$ model. Each integral over the auxiliary field $\sigma(\tilde{x})$ on the dual site $\tilde{x}$ gives,

$$
I_{\tilde{x}}=\int d \sigma(\tilde{x}) \mathrm{e}^{-S_{A F}-\frac{\sigma(\tilde{x})}{8}\left(\sum_{i,[x, \tilde{l}} \bar{x}_{i}(x) \chi_{i}(x)\right)}=\mathscr{N} \mathrm{e}^{-S_{I}(\tilde{x})},
$$

where $\mathscr{N}=\sqrt{2 \pi g^{2} / N}$ and

$$
S_{I}(\tilde{x})=-\frac{g^{2}}{128 N}\left[\sum_{i,[x, \tilde{x}]} \bar{\chi}_{i}(x) \chi_{i}(x)\right]^{2},
$$

is the effective four-fermion interaction term at each dual site $\tilde{x}$. The symbol $[x, \tilde{x}]$ denotes the set of all lattice sites surrounding the dual site $\tilde{x}$. Thus, each integral generates many four-fermion couplings of the form $\bar{\chi}_{i}(x) \chi_{i}(x) \bar{\chi}_{j}(y) \chi_{j}(y)$ where $i$ and $j$ are arbitrary flavor indices and $x$ and $y$ are corners of the cube surrounding the dual site $\tilde{x}$. We can classify the possible couplings into four types based on the bonds $\langle x y\rangle$ connecting the corners $x$ and $y$. If the two corners are the same we refer to it as a site-bond or a $S$-bond. If the two corners are the two neighboring sites we get a $L$-bond (or a link-bond). Similarly, if the two corners are across a face diagonal or a body diagonal, we call the bonds $F$-bond and $B$-bond respectively. These four bond types are illustrated Fig. 2.
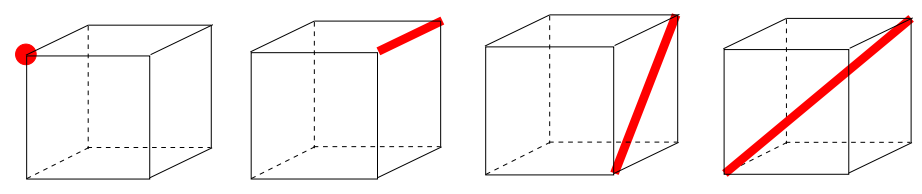

Figure 2: An illustration of the four types of four-fermion couplings (or bonds) generated through the auxiliary field integration. From left to right we have a $S, L, F$ and $B$ bond respectively.

Integration over all the auxiliary field variables yields the four-fermion interaction term of the action $S_{Z_{2} \text {,int }}=\sum_{\tilde{x}} S_{I}(\tilde{x})$. Collecting the terms in each of the four types of four fermion couplings separately we see that

$$
S_{Z_{2}, \text { int }}=U_{S} \mathscr{B}_{S}+U_{L} \mathscr{B}_{L}+U_{F} \mathscr{B}_{F}+U_{B} \mathscr{B}_{B}
$$

where $U_{S} / 4=U_{L} / 4=U_{F} / 2=U_{B}=g^{2} /(64 N)$ and

$$
\mathscr{B}_{\text {bond }}=\sum_{i, j,\langle x y\rangle \in \text { bond }} \bar{\chi}_{i}(x) \chi_{i}(x) \bar{\chi}_{j}(y) \chi_{j}(y) \text {. }
$$

Based on the above results, the partition function of the $Z_{2}$ model can be rewritten as

$$
Z_{Z_{2}}=\int \prod_{i}\left[d \bar{\chi}_{i} d \chi_{i}\right] \mathrm{e}^{-S_{Z_{2}}}
$$


where $S_{Z_{2}}=S_{0}+S_{Z_{2}, \text { int }}$ is the equivalent four-fermion action of the model. $S_{0}=\sum_{x, y, i} \bar{\chi}_{i}(x) D_{x, y} \chi_{i}(y)$ is the free fermion action.

In the fermion bag approach, each four-fermion coupling is represented as a bond. For example the four-fermion coupling of the type $\bar{\chi}_{i}\left(x_{p}\right) \chi_{i}\left(x_{p}\right) \bar{\chi}_{j}\left(x_{q}\right) \chi_{j}\left(x_{q}\right)$ can be denoted by the bond variable $b_{i j}\left(x_{p}, x_{q}\right)=0,1$, such that if it is 0 then no bond is assumed to exist between the sites $x_{p}$ and $x_{q}$, otherwise the specific four-fermion coupling is inserted in the partition function. Due to the Grassmann nature of the couplings higher powers of the couplings do not exist. More details can be found in [10]. Thus, in the fermion bag formulation, the partition function can be written as a sum over these bond configurations $[b]$, such that

$$
\begin{aligned}
Z_{Z_{2}} & =\sum_{[b]} U_{S}^{n_{S}} U_{L}^{n_{L}} U_{F}^{n_{F}} U_{B}^{n_{B}} \int \prod_{i}\left[d \bar{\chi}_{i} d \chi_{i}\right] \mathrm{e}^{-S_{0}} \prod_{i} \bar{\chi}_{i}\left(x_{i_{1}}\right) \chi_{i}\left(x_{i_{2}}\right) \ldots \bar{\chi}_{i}\left(x_{i_{k_{i}}}\right) \chi_{i}\left(x_{i_{k_{i}}}\right) \\
& =\sum_{[b]} U_{S}^{n_{S}} U_{L}^{n_{L}} U_{F}^{n_{F}} U_{B}^{n_{B}}\left\{\prod_{i} C_{i}\left(x_{i_{1}}, \ldots, x_{i_{k_{i}}}\right)\right\}
\end{aligned}
$$

where $n_{S}, n_{L}, n_{F}$ and $n_{B}$ are the total number of bonds of each type and the correlation function $C_{i}\left(x_{i_{1}}, . ., x_{i_{k_{i}}}\right)$ was defined in Eq.(3.1). A given bond configuration $[b]$ uniquely determines the $k_{i}$ sites $x_{i_{1}} \ldots x_{i_{k_{i}}}$ (ordered in a consistent way). Since we argued above that $C_{i}\left(x_{i_{1}}, \ldots, x_{i_{k_{i}}}\right) \geq 0$ there is no sign problem in this expansion of the partition function for all non-negative values of $U_{S}, U_{L}, U_{F}$, $U_{B}$, any positive integer $N$ and real mass $m$.

In the case of the $U(1)$ model, we need to integrate over both the auxiliary fields $\sigma(\tilde{x}), \pi(\tilde{x})$ on every dual site. It is straightforward to verify that

$$
I_{\tilde{x}}=\int[d \sigma(\tilde{x}) d \pi(\tilde{x})] \mathrm{e}^{-S_{A F}-\frac{\sigma(\tilde{x})}{8}\left(\sum_{i,[\tilde{x},]} \bar{\chi}_{i}(x) \chi_{i}(x)\right)} \times \mathrm{e}^{-i \frac{\pi(\tilde{x})}{8}\left(\sum_{i,[\tilde{x},]} \varepsilon(x) \bar{\chi}_{i}(x) \chi_{i}(x)\right)}=\mathscr{N} \mathrm{e}^{-S_{I}(\tilde{x})}
$$

where $\mathscr{N}=\left(4 \pi g^{2} / N\right)$ and

$$
S_{I}(\tilde{x})=\frac{g^{2}}{64 N}\left\{\left[\sum_{i,[x, \tilde{x}]} \bar{\chi}_{i}(x) \chi_{i}(x)\right]^{2}-\left[\sum_{i,[x, \tilde{x}]} \varepsilon(x) \bar{\chi}_{i}(x) \chi_{i}(x)\right]^{2}\right\},
$$

Interestingly, the four-fermion couplings of the type $S$ and $F$ get canceled between the two terms in the above equation. On the other hand couplings of the type $L$ and $B$ survive so that the four-fermion action for the $U(1)$ model turns out to be

$$
S_{U(1)}=S_{0}+U_{L} \mathscr{B}_{L}+U_{B} \mathscr{B}_{B}
$$

with $U_{L} / 4=U_{B}=g^{2} /(16 N)$. Thus, the only difference between the $Z_{2}$ and $U(1)$ models is that the couplings $U_{S}=U_{F}=0$ in the $U(1)$ model. Indeed these couplings break the $U(1)$ symmetry to a $Z_{2}$ symmetry as can be easily verified. Since we already proved that the sign problem in the $Z_{2}$ model was absent for all non-negative values of $U_{S}, U_{L}, U_{F}, U_{B}$ and $N$ in the fermion bag formulation, the same is true for the $U(1)$ model as well.

\section{Conclusions}

The fermion bag approach provides an alternative approach to fermion field theories where solutions to new sign problems emerge naturally. Here we have demonstrated that some sign problems in the auxiliary field formulation of GN models, especially with $Z_{2}$ and $U(1)$ chiral symmetries, disappear in the fermion bag approach. While we have not shown here, we can solve sign 
problems in some lattice field theories containing both dynamical boson and fermion fields with similar chiral symmetries. In these more complex models, the solutions emerge when bosons are formulated in the world-line approach and the fermions are formulated in the bag approach. Such an approach to quantum field theories was proposed in [16].

Sign problems in other fermion models with more complex symmetries are also solvable in the fermion bag approach. However, in many interesting cases the Boltzmann weight of a fermion bag, although non-negative, turns out to be a fermionant instead of a determinant [17]. Since the computation of the fermionant can be exponentially hard, the fermion bag approach loses its practical appeal in such cases. Still, we believe that there are many other interesting models where the weight of the fermion bag continues to be positive and computable with polynomial effort.

\section{Acknowledgments}

I would like to thank S. Chandrasekharan for his collaboration and many fruitful discussions. This work was supported in part by the Department of Energy grants DE-FG02-05ER41368 and DE-FG02-00ER41132.

\section{References}

[1] M. Franz and Z. Tesanovic, Phys. Rev. Lett. 87, 257003 (2001).

[2] Y. Ran, M. Hermele, P. A. Lee, and X.-G. Wen, Phys. Rev. Lett. 98, 117205 (2007).

[3] P. F. Bedaque and U. van Kolck, Ann.Rev.Nucl.Part.Sci. 52, 339 (2002), nucl-th/0203055.

[4] E. Epelbaum, H. Krebs, D. Lee, and U.-G. Meissner, Phys.Rev.Lett. 104, 142501 (2010).

[5] B. Rosenstein, B. Warr, and S. Park, Phys.Rept. 205, 59 (1991).

[6] I. F. Herbut, V. Juričić, and B. Roy, Phys. Rev. B 79, 085116 (2009).

[7] W. Armour, S. Hands, and C. Strouthos, Phys.Rev. B84, 075123 (2011).

[8] D. Grabowska, D. B. Kaplan, and A. N. Nicholson, (2012), 1208.5760.

[9] S. Hands, A. Kocic, and J. B. Kogut, Annals of Physics 224, 29 (1993).

[10] S. Chandrasekharan, Phys.Rev. D82, 025007 (2010), 0910.5736.

[11] S. Chandrasekharan and A. Li, PoS LATTICE2011, 058 (2011), 1111.5276.

[12] S. Chandrasekharan and A. Li, Phys. Rev. Lett. 108, 140404 (2012), 1111.7204.

[13] S. Chandrasekharan and A. Li, Phys.Rev. D85, 091502 (2012), 1202.6572.

[14] S. Chandrasekharan, Phys.Rev. D86, 021701 (2012), 1205.0084.

[15] S. Chandrasekharan and U.-J. Wiese, Phys. Rev. Lett. 83, 3116 (1999).

[16] S. Chandrasekharan, PoS LATTICE2008, 003 (2008).

[17] S. Chandrasekharan and U.-J. Wiese, (2011), 1108.2461. 